\title{
Reliability of Tissue Microarrays in Detecting Protein Expression and Gene Amplification in Breast Cancer
}

\author{
DaoHai Zhang, Ph.D., Manuel Salto-Tellez, M.D., Thomas Choudary Putti, M.D., Elaine Do, B.Sc., \\ Evelyn Siew-Chuan Koay, Ph.D. \\ Molecular Diagnosis Centre (DHZ, MS-T, ED, ES-CK), National University Hospital, and Department of \\ Pathology (DHZ, MS-T, TCP, ED, ES-CK), National University of Singapore, Singapore
}

Tissue microarrays allow high throughput molecular profiling of diagnostic or predictive markers in cancer specimens and rapid validation of novel potential candidates identified from genomic and proteomic analyses in a large number of tumor samples. To validate the use of tissue microarray technology for all the main biomarkers routinely used to decide breast cancer prognostication and postsurgical adjuvant therapy, we constructed a tissue microarray from 97 breast tumors, with a single $0.6 \mathrm{~mm}$ core per specimen. Immunostaining of tissue microarray sections and conventional full sections of each tumor were performed using wellcharacterized prognostic markers (estrogen receptor ER, progesterone receptor $P R$ and c-erbB2). The full section versus tissue microarray concordance for these stains was $\mathbf{9 7 \%}$ for $\mathrm{ER}, \mathbf{9 8 \%}$ for $\mathrm{PR}$, and $\mathbf{9 7 \%}$ for c-erbB2, respectively, with a strong statistical association (kappa value more than 0.90 ). Fluorescence in situ hybridization analysis for HER-2/ neu gene amplification from the single-core tissue microarray was technically successful in about $90 \%(87 / 97)$ of the cases, with a concordance of 95\% compared with parallel analyses with the full sections. The correlation with other pathological parameters was not significantly different between full-section and array-based results. It is concluded that the constructed tissue microarray with a single core per specimen ensures full biological representativeness to identify the associations between biomarkers and clinicopathological parameters, with no significant associated sampling bias.

Copyright ( $\odot 2003$ by The United States and Canadian Academy of Pathology, Inc.

VOL. 16, NO. 1, P. 79, 2003 Printed in the U.S.A.

October 28, 2002.

Address reprint requests to: Manuel Salto-Tellez, M.D., Department of Pathology, National University of Singapore, 5 Lower Kent Ridge Road, Singapore 119074; e-mail: patmst@nus.edu.sg; fax: 65-6778-0671.

DOI: 10.1097/01.MP.0000047307.96344.93
KEY WORDS: Breast tumors, Fluorescence in situ hybridization, Immunohistochemistry, Tissue microarrays.

Mod Pathol 2003;16(1):79-85

Both genetic and environmental factors play a key role in the development of breast cancer, one of the commonest cancers in the world. Understanding the basis of tumor development and progression, and identifying biomarkers for assessment of prognosis and prediction of therapy outcome are integral parts of current research efforts. Traditionally, three wellcharacterized biomarkers-estrogen receptor or ER (1-4), progesterone receptor or PR $(3,4)$, and c-erbB2 or HER-2/neu oncogene (5-7)—have been used in the clinical analysis of breast cancer by immunohistochemistry and fluorescence in situ hybridization (FISH), both applied to full sections of formalin-fixed, paraffin-embedded tumor tissues. However, this would be time-consuming and tedious when processing large numbers of tumors or when screening with multiple markers. The recently developed tissue microarray, composed from multiple donor tumors systematically aligned within a single recipient block, allows for high throughput molecular profiling of many tumor tissues simultaneously in a single experiment. Thin sections cut from such tissue microarray blocks can be used to study gene amplification and protein overexpression by DNA and RNA in situ hybridization or by immunohistochemistry, with tremendous savings in analysis time, labor and reagent costs. This technology has the potential to significantly accelerate studies seeking for the association between molecular changes and clinical endpoints (8, 9) and the validation of novel biomarkers (10). The high speed of arraying, the lack of any significant damage to the donor blocks, and the uniform treatment and assessment of regularly arrayed tumors representing different tumor stages, histological grades, and histological subtypes are the most significant advantages of the tissue microarray technology. Use of tissue microarrays will greatly accelerate the 
transition of basic research findings to clinical applications.

One of the limitations of the tissue microarray technology is that the "punched" minute samples from donor tissues may not always be representative of the entire tumors with their well-established heterogeneity. Therefore, "punching" from multiple sites from each original block was recommended to reduce the sampling problem (11-13). However, it was reported in a recent study that a single "punch" uncovered most of the information on ER expression in breast cancer, and that tumor heterogeneity did not negatively influence the predictive power of tissue microarray results (9). The associations between molecular changes and clinical endpoints detected on tissue microarrays based on the single core specimen per tumor suggest that biomarkers with prognostic significance are more likely to be expressed in a relatively homogeneous manner in the tumor. The more homogeneously a marker of interest is expressed in the tumor and the more histologically homogeneous the tumor is, the more likely the tissue microarray data will reproduce the findings from the full sections. Sampling a typical and representative region of the tumor is certainly the key step in tissue microarray construction. Therefore, careful selection of representative regions of tumors and precise "punching" from donor tissues can reproduce sufficient information in cell populations.

In the present study, we investigated the efficiency and representativeness of immunohistochemistry and FISH analyses in our constructed tissue microarrays of breast cancer. We constructed a tissue microarray with a single $0.6 \mathrm{~mm}$ core biopsy per specimen and applied the most commonly used diagnostic and prognostic markers, including monoclonal antibodies against ER, PR and c-erbB2 by immunohistochemistry and the locus-specific HER-2/ neu probe by FISH. We defined the concordance of data from both tissue microarray and full sections and the impact of data discrepancy between the two methods with regard to the correlation with other pathological parameters.

\section{MATERIALS AND METHODS}

\section{Patients}

Ninety-seven breast cancer samples from the National University Hospital of Singapore were included in this study. The age ranged from 32 to 86 years, with an average of 53 years. The tumors included invasive ductal carcinoma $(n=84)$, ductal carcinoma in situ $(n=7)$, medullary carcinoma ( $n$ $=3$ ) and mucinous carcinoma $(n=3)$. Forty-six cases had metastases to lymph nodes while 51 were node-free. Among the 97 tumors, $11.3 \%(n=11)$ had a histologic grade $1,39.2 \%(n=38)$ a grade 2 , and $49.5 \%(n=48)$ a grade 3 .

\section{Tissue Microarray Construction}

A total of 194 formalin-fixed, paraffin-embedded breast tissue samples (97 tumors and 97 normal) were used for tissue microarray construction, as described by Kononen et al. (9). Ninety-seven tumors, with 5 normal tissues as internal negative controls, were arrayed in one paraffin block and 97 normal tissues, in another. Before arraying, $4-\mu \mathrm{m}$ sections from each tissue block were stained with Hematoxylin and Eosin (H\&E) and a morphologically representative area of tumor was defined by the pathologists. Tissue cylinders with a diameter of $0.6 \mathrm{~mm}$ were then punched from the targeted tumor area of each donor tissue block and deposited into a recipient block using a tissue arraying instrument (Beecher Instruments ${ }^{\circledR}$, Silver Spring, MD). After construction of the array block, multiple consecutive $4-\mu \mathrm{m}$ sections were cut until all the 97 tissue samples were represented on a single section. One such section was placed on a microscopic slide and H\&E-stained for histological verification of the adequacy of the arrayed tumor tissues. The consecutive sections were separately placed on charged polylysine-coated slides for immunohistochemistry and FISH analyses. Sections from the second tissue microarray of normal samples were prepared by the same process and served as controls.

\section{Immunohistochemistry}

Standard indirect immunoperoxidase procedures were used for immuno-histochemistry. Sections from formalin-fixed, paraffin-embedded tissues were deparaffinized, treated with $3 \%$ hydrogen peroxide in phosphate-buffered saline and pretreated at $95^{\circ} \mathrm{C}$ for 10 minutes in $10 \mu \mathrm{mol} / \mathrm{L}$ citrate buffer (pH 6.0). Three monoclonal antibodies were used for detection of ER (ER1D5, 1:1000), PR (NCL-PGR, 1A6, 1:1000) and c-erbB2 (HercepTest ${ }^{\mathrm{TM}}$ K5204, 1:500), all from $\mathrm{DAKO}^{\circledR}$. Diaminobenzidine was used as the chromogen and hematoxylin as the nuclear counterstain. The same scoring criteria were applied to the tissue microarray and the large sections. All slides were independently analyzed by the pathologists (MST and TCP) and the principal researcher (ZDH). Tumors were considered positive for ER and PR if more than $10 \%$ of tumor cells showed an unequivocal nuclear staining. Tumors exhibiting definite cytoplasmic membrane staining (grade $2+$ and $3+$ ) were considered to be positive for c-erbB2 expression.

\section{Fluorescence In Situ Hybridization (FISH)}

The tissue array sections were treated according to the Paraffin Pretreatment Reagent Kit $\left(\mathrm{Vysis}^{\circledR}\right)$ proto- 
col, before hybridization. FISH was performed with a locus-specific Spectrum Orange-labeled HER-2/neu probe and a Spectrum Green-labeled chromosome 17 centromeric probe (CEP-17) as an internal control reference probe. Hybridization and posthybridization washes were carried out according to the LSI HER-2/ neu kit $\left(\right.$ Vysis $\left.^{\circledR}\right)$ procedure. Briefly, the deparaffinized slides were immersed in $0.2 \mathrm{~mol} / \mathrm{L} \mathrm{HCl}$ for 20 minutes and after washing with water and Wash Solution, were incubated in $1 \mathrm{~mol} / \mathrm{L}$ sodium thiocyanate solution at $80^{\circ} \mathrm{C}$ for 30 minutes. The pretreated slides were further treated with protease solution $(0.5 \mathrm{mg} / \mathrm{mL}$ in $0.9 \% \mathrm{NaCl}, \mathrm{Vysis}^{\circledR}$ ) for 20 minutes at $37^{\circ} \mathrm{C}$, fixed in $10 \%$ buffered formalin for 10 minutes, air-dried and denatured for 5 minutes. The probe mixture was applied to each slide, with minimal delay. After hybridization at $37^{\circ} \mathrm{C}$ overnight, the sections were washed and counterstained with $0.2 \mu \mathrm{mol} / \mathrm{L}$ DAPI $(4,6-$ diamidino-2-phenylindole). The fluorescent signals were scored with an Olympus fluorescence microscope equipped with double-bandpass filters for simultaneous visualization of Spectrum Green and Spectrum Orange signals. The relative ratio of the orange HER-2/ neu gene signals to the green chromosome 17 centromeric signals was evaluated by the cytogeneticist (ED), with a ratio of $\geq 2: 1$ arbitrarily defined as evidence for HER-2/neu gene amplification.

\section{Statistical Analysis}

The association of the results obtained with the full sections and those with the tissue microarray sections was studied using kappa statistics (14). A kappa value more than 0.5 was considered to denote a strong association between the two sets of results. Contingency table analysis and $\chi^{2}$ test were used to analyze the correlations of immunohistochemistry and FISH in full sections and tissue microarray with other pathological parameters. The survival curves were not described as all the cases were diagnosed in the years 2000 and 2001.

\section{RESULTS}

An overview of the results we obtained is presented in Table 1.

\section{Efficiency of Biopsy Arraying}

We found only 4 single-core samples missing from the H\&E-stained section prepared from the tumor tissue microarray block containing 102 representative core biopsies, including 5 from normal tissues as controls. The remaining 98 samples, including the 5 normal tissues, were verified as representative core biopsies of their respective donor tumors by the pathologists (MST and TCP). The rate of nonrepresentation for the single-core array was $4 \%$, giving an efficiency of $96 \%$ for the 97 tumor samples. The next three consecutive sections were used for ER, PR and c-erbB2 immunohistochemistry analyses. Two other consecutive sections were prepared for H\&E examination and HER-2/neu FISH analysis. Representative positive single-core results for both immunohistochemistry and FISH are shown in Figure 1.

\section{Estrogen Receptor (ER) Overexpression}

Ninety-three of the 97 tumor cases $(91.9 \%)$ on the tissue microarray were accessible for ER analysis, with 53 positive cases (57\%) showing nuclear staining in more than $10 \%$ of the tumor nuclei and 40 negative cases (40\%). In comparison, for the full sections of the studied tumors, 56 cases (60.2\%) were read as positive and 37 cases $(39.8 \%)$ as negative. Overall, a concordance of $97 \%$ among the accessible cases and a strong statistical association (kappa value, $\mathrm{k}=0.93$ ) were found.

\section{Progesterone Receptor (PR) Overexpression}

Of the 93 tumor cores in the tissue microarray, 54 stained positive $(58.1 \%)$. For the full sections, 56 cases $(60.2 \%)$ were defined as positive. The two mismatches were negative in tissue microarray and positive in the full sections. A strong statistical association between the two methods for the PR analysis was also demonstrated (kappa value, $\mathrm{k}=0.95$ ).

\section{c-erbB2 Overexpression}

In the tissue microarray section, 5 tumor samples were missed during staining (technical error) and only the remaining 92 tumor cases were available

TABLE 1. Comparison between Full Sections and Arrayed Sections of Estrogen Receptor (ER), Progesterone Receptor (PR), c-erbB2, and HER-2/neu Status in Breast Tumors

\begin{tabular}{|c|c|c|c|c|c|}
\hline & & $\begin{array}{c}\text { Full } \\
\text { Sections }\end{array}$ & $\begin{array}{l}\text { Arrayed } \\
\text { Sections }\end{array}$ & Concordance (\%) & $\kappa$-value ${ }^{\mathrm{a}}$ \\
\hline \multirow[t]{2}{*}{ ER } & + & 56 & 53 & $97(n=93)$ & 0.93 \\
\hline & - & 37 & 40 & & \\
\hline \multirow[t]{2}{*}{ PR } & + & 56 & 54 & $98(n=93)$ & 0.95 \\
\hline & - & 37 & 39 & & \\
\hline \multirow[t]{2}{*}{ c-erbB2 } & + & 33 & 30 & $97(n=92)$ & 0.92 \\
\hline & - & 59 & 62 & & \\
\hline \multirow[t]{2}{*}{ HER-2/neu } & + & 23 & 20 & $95(n=87)$ & 0.89 \\
\hline & - & 64 & 67 & & \\
\hline
\end{tabular}

${ }^{a}$ Kappa coefficient (14). 
for immunohistochemistry analysis. The c-erbB2 overexpression was noted in 30 cases $(32.6 \%)$ while 61 cases $(66.3 \%)$ were negative. In comparison, for c-erbB2 expression in the full sections, 33 of the 92 cases $(34.8 \%)$ showed positivity in the full sections, with 3 cases demonstrating discrepancy, giving a non-concordance rate of $3 \%$ and a highly significant statistical association between the methods (kappa value, $\mathrm{k}=0.92$ ).

\section{HER-2/neu Gene Amplification by FISH}

HER-2/ neu gene amplification was only scored to be present when the copy number of HER-2 gene signals exceeded that of the chromosome 17 centromeric probe (CEP-17) by at least 2 -fold in $10 \%$ or more of the tumor cells. FISH analysis was success- ful in 87 of the 97 arrayed tumor samples (3 samples were lost and 7 others were non-informative). HER$2 /$ neu gene amplification was detected in 20 of the 87 interpretable cases. An example of the HER-2 gene amplification by FISH in the tissue microarray section is shown in Figure 1. When matched against the corresponding FISH data from the full sections, three cases showed discrepancy. The nonconcordance rate of $4.5 \%$ and the kappa value of 0.89 suggest a very good agreement of the FISH results from the two methods.

\section{Correlation with Other Pathological Parameters}

To assess the similarity (or lack thereof) of overexpression of biomarkers in relation to other pathological parameters, we independently analyzed
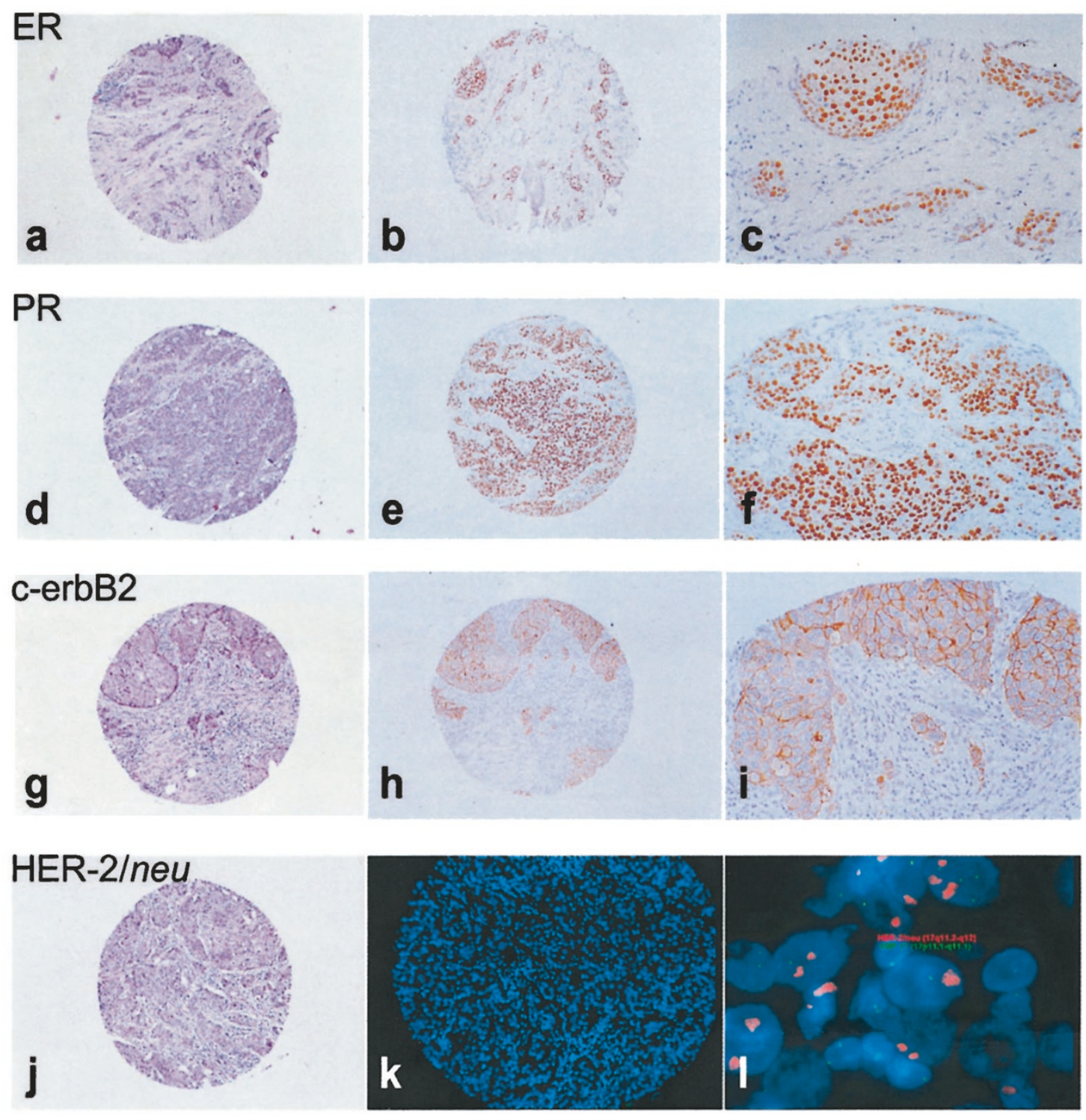

FIGURE 1. Representative examples of ER, PR, and c-erbB2 immunohistochemistry and HER-2/neu gene amplification (FISH) in breast tumor tissue arrays. a, d, $\mathbf{g}$, and $\mathbf{j}$, H\&E staining of four tissue samples $(\times 42)$; $\mathbf{b}, \mathbf{e}$, and $\mathbf{h}$, immunostaining $(\times 42)$; $\mathbf{c}$, $\mathbf{f}$, and $\mathbf{i}$, enlarged immunostaining of the specific area $(\times 200)$; $\mathbf{k}$, a tissue microarray sample for FISH analysis $(\times 100)$; $\mathbf{l}$, HER-2/neu gene amplification $(\times 1000)$ : cells showing a massive increase in the number of the orange signals as compared with the green internal control signals. 
both data sets generated from tissue microarray and full sections using the contingency table analysis and the $\chi^{2}$ test. From the data of the full sections, both ER and PR expressions were significantly correlated to the histological tumor grade $(P$ $<.001)$. The c-erbB2 expression was also strongly associated with the tumor grade $(0.001<P<.01)$, but in an inverse manner. A similar statistical association was noted with the tissue microarray data, including a strong relationship of ER, PR and c-erbB2 with tumor grade $(P<.001)$. HER-2/neu amplification data from both methods showed a similar statistical association with the histological tumor grade $(0.001<P<.01)$. Our analysis indicated that the single-core tissue array-derived data are comparable with the data from full sections in correlating with other pathological parameters.

\section{DISCUSSION}

Tissue microarrays constructed from multiple donor tumors are a potentially useful tool for rapid and efficient analysis of large numbers of paraffinembedded tissue (9), facilitating standardized analysis of multiple gene expression and amplification in the same tumor or the same tumor site, using the same kind of probes and standardized interpretation criteria. The main advantages of tissue microarray technology include the improved analytical capacity, the negligible damage caused to the original tissue blocks, and the precise positioning of tissue specimens on the recipient block. Usually, tissue core "punches" of 0.6 to $2.0 \mathrm{~mm}$ in diameter are taken from representative areas of paraffinembedded tissues and are arrayed on a single recipient block. These punched-out cores are considered to be large enough to enable adequate histomorphology assessment and analysis of biomarker expression and amplification at the DNA, RNA and protein levels (15-18). Data from three recent studies further suggest that the minute tissue samples in an array format are sufficiently representative of their donor tumors to allow authentic association studies between molecular alterations and clinical endpoints $(8,17,19,20)$. However, the tissue microarray technique is limited by the risk of non-representative sampling of the potentially heterogeneous tumors, which are often evident both at the morphological and genetic levels (21-23). Thus, the issues of what is the minimum number of "punches" per case and up to what extent the tissue microarray data can reproduce large section data are important when analyzing the clinicopathological associations. Hoos et al. (11) reported that triplicate cores offered a high rate of assessable cases and a lower rate of non-concordant readings than one or two cores, whereas Rubin et al. (13) demon- strated that four cores was optimal to represent tumor protein expression. However, this would reduce the number of tumor samples that can be assembled in a recipient block and render the process somewhat time-consuming and tedious. To maximize the efficiency of the tissue microarray technology, it is crucial to identify the minimum number of cores needed for meaningful results, to carefully select representative areas on H\&Estained full sections based on the tumor morphology and to precisely transfer the target site from the donor to the recipient blocks. We have successfully constructed a single-core tissue microarray of breast cancer wherein 93 of the 97 arrayed tumor biopsies were fully representative of the donor tissues. The current success rate in our laboratory for arraying breast cancers is greater than $95 \%$, with loss of accessible cases limited to only $4 \%$.

All the biomarkers analyzed in this study are the ones routinely used for breast cancer prognostication and stratification for further therapy. To identify the concordance of the immunohistochemical expression profiles of the tissue array containing a single core per specimen and the full sections, we used well-characterized antibodies against ER, PR and c-erbB2, and obtained concordance rates of $97 \%, 98 \%$ and $97 \%$, respectively. Our result for PR expression was higher than the $88 \%$ concordance reported by Torhorst et al. (8). The few discrepant readings that we found in ER, PR and c-erbB2 staining changed the original abnormal results found in full sections to normal results in arrayed tissues and hence resulted in slightly lower ratios of abnormal expression patterns in tissue microarray. Full section analysis resulted in $60.2 \%, 60.2 \%$ and $34.8 \%$ of cases with abnormal expression of ER, PR and c-erbB2, respectively. Corresponding respective readings on the single-core tissue microarray were $57 \%, 58 \%$ and $32.6 \%$. The high concordance and the strong agreement (kappa value more than 0.90) between the two methods suggest that tissue microarray can accurately reflect immunohistochemistry information currently derived from full sections.

The FISH technique has been successfully used for analysis of gene amplification by high throughput tissue microarray $(16,18,24,25)$. To validate the FISH analysis in our tissue microarray, we performed HER$2 /$ neu gene amplification analysis and compared the data obtained with the original full-section FISH data. Seven non-interpretable samples were associated with various technical problems of FISH, such as weak hybridization signals or excessive background. The technical adequacy (87 of 97 tissues or $90 \%$ ) is higher than the results (75 85\%) from Schraml et al. (18) and comparable with data (95.1\%) from four repeats reported by Simon et al. (25). Well-defined hybridization signals were observed in $23 \%$ of the 87 accessible samples in tissue microarray, producing a 
95\% agreement with the data from full sections and a statistically strong association (kappa value: 0.89). However, when we used the $\chi^{2}$ test to compare the strength of association of the biomarker data from full sections and tissue microarray with the other pathological parameters, we did not find any significant differences between the two methods, with both showing strong associations with the other pathological parameters.

Our study included all the routinely-used biomarkers to decide patient prognosis and adjuvant therapy in breast cancer, and our data demonstrated the high reliability and comparability of tissue microarraybased immunohistochemistry and FISH using the same cut-off values for full section analysis. The biopsy of $0.6 \mathrm{~mm}$ diameter taken from a typical and representative region of the tumor provides a reliable and efficient system for large-scale analysis of cancer tissues on the tissue microarray platform and is useful for large-scale clinicopathological studies, conserving the molecular profile of the molecular markers that are clinically relevant.

Acknowledgments: This work was funded by a grant from the National Health Group of Singapore, NHGRPR/01121. DHZ and ED are funded by a grant from The Health Service Development Program, Ministry of Health, Singapore. The skillful technical assistance of Mr. Ow Cheok Kee and Mr. Tan Tee Chok is greatly appreciated.

\section{REFERENCES}

1. Robertson JF, Bates K, Pearson D, Blarney RW, Nicholson RI. Comparison of two estrogen receptor assays in the prediction of the clinical course of patients with advanced breast cancer. J Clin Oncol 1992;65:727-30.

2. DeSombre ER, Thorpe SM, Rose C, Blough RR, Andersen $\mathrm{KW}$, Rasmissen BB, et al. Prognostic usefulness of estrogen receptor immunohistochemical assays for human breast cancer. Cancer Res 1986;46:4256s-64s.

3. Nizzoli R, Bozzetti C, Savoldi L, Manotti L, Naldi N, Camisa $\mathrm{R}$, et al. Immunocytochemical assay of estrogen and progesterone receptors in fine needle aspirates from breast cancer patients. Acta Cytol 1994;38:933-8.

4. Pertschuk LP, Kim DS, Nayer K, Feldman JG, Eisenberg KB, Carter AC, et al. Immunocytochemical estrogen and progestin receptor assays in breast cancer with monoclonal antibodies: histopathologic, demographic, and biochemical correlations and relations to endocrine response and survival. Cancer 1990;66:1663-70.

5. Tsutsui S, Ohno S, Murakami S, Hachitanda Y, Oda S. Prognostic value of c-erbB2 expression in breast cancer. J Surg Oncol 2002;79:216-23.

6. Andrulis IL, Bull SB, Blakstein ME, Sutherland D, Mak C, Sidlofsky S, et al. neu/erbB2 amplification identifies a poorprognosis group of women with node-negative breast cancer. J Clin Oncol 1998;16:1340-9.

7. Revillion F, Bonneterre J, Peyrat JP. ERBB2 oncogene in human breast cancer and its clinical significance. Eur J Cancer 1998;34:791-808.
8. Torhorst J, Bucher C, Kononen J, Haas P, Zuber M, Kochli OR, et al. Tissue microarrays for rapid linking of molecular changes to clinical endpoint. Am J Pathol 2001;159:224956.

9. Kononen J, Bubendorf L, Kallioniemi A, Barlund M, Schraml $\mathrm{P}$, Leighton S, et al. Tissue microarrays for high-throughput molecular profiling of tumor specimens. Nat Med 1998;4: 844-7.

10. Rubin MA, Zhou M, Dhanasekaran SM, Varambally S, Barrette T, Sanda MG, et al. $\alpha$-methylacyl coenzyme. A racemase as a tissue biomarker for prostate cancer. J Am Med Assoc 2002;287:1662-70.

11. Hoos A, Urist MJ, Stojadinovic A, Mastorides S, Dudas ME, Leung DHY,et al. Validation of tissue microarrays for immunohistochemical profiling of cancer specimens using the example of human fibroblastic tumors. Am J Pathol 2001; 158:1245-51.

12. Mucci NR, Akdas G, Manely S, Rubin MA. Neuroendocrine expression in metastatic prostate cancer: evaluation of high throughput tissue microarrays to detect heterogeneous protein expression. Hum Pathol 2000;31:406-14.

13. Rubin MA, Dunn R, Strawderman M, Pienta K. Tissue microarray sampling strategy for prostate cancer biomarker analysis. Am J Surg Pathol 2002;26:312-9.

14. Altman DG. Practical statistics for medical research. London: Chapman and Hall; 1996;404-8.

15. Ristimaki A, Sivula A, Lundin J, Lundin M, Salminen T, Haglund C, et al. Prognostic significance of elevated cyclooxygenase-2 expression in breast cancer. Cancer Res 2002;62:632-5.

16. Bubendorf L, Kononen J, Kivisto P, Schraml P, Moch H, Gasser TC, et al. Survey of gene amplifications during prostate cancer progression by high throughput fluorescence in situ hybridization on tissue microarrays. Cancer Res 1999;59:803-6.

17. Richter J, Wagner U, Kononen J, Fijan A, Bruderer J, Schmid $\mathrm{U}$, et al. High-throughput tissue microarray analysis of cyclin E gene amplification and overexpression in urinary bladder cancer. Am J Pathol 2000;157:787-94.

18. Schraml P, Konone J, Bubendorf L, Moch H, Bissig H, Nocito A, et al. Tissue microarrays for gene amplification surveys in many different tumor types. Clin Cancer Res 1999;5:196675.

19. Chung GG, Provost E, Kielhorn EP, Charette LA, Smith BL, Rimm DL. Tissue microarray analysis of $\beta$-catenin in colorectal cancer shows nuclear phospho- $\beta$-catenin is associated with a better prognosis. Clin Cancer Res 2001;7:4013-20.

20. Xie W, Mertens JC, Reiss DJ, Rimm DL, Camp RL, Haffty BG, et al. Alterations of Smad signaling in human breast carcinoma are associated with poor outcome: a tissue microarray study. Cancer Res 2002;62:497-505.

21. Allred DC, Harvey JM, Berardo M, Clasrk GM. Prognostic and predictive factors in breast cancer by immunohistochemical analysis. Mod Pathol 1998;11:155-68.

22. Bergers E, van Diest PJ, Baak JP. Tumor heterogeneity of DNA cell cycle variables in breast cancer measured by flow cytometry. J Clin Pathol 1996;49:931-7.

23. Braun S, Hepp F, Sommer HL, Pantel K. Tumor antigen heterogeneity of disseminated breast cancer cells: implications for immunotherapy of minimal residual disease. Int J Cancer 1999;84:1-5.

24. Rummukainen JK, Salminen T, Lundin J, Joensuu H, Isola JJ. Amplification of c-myc oncogene by chromogenic and fluorescence in situ hybridization in archival breast cancer tissue array samples. Lab Invest 2001;81:1545-51.

25. Simon R, Nocito A, Hubscher T, Bucher C, Torhorst J, Schraml P, et al. Patterns of HER-2/neu amplification and overexpression in primary and metastatic breast cancer. J Natl Cancer Inst 2001;93:1141-6. 


\section{Book Review}

\section{Kumar V, Cotran RS, Robbins SL: Robbins Basic Pathology, 873 pp, Philadelphia, W.B. Saunders, 2003 (\$62.95).}

It is my great privilege to review the seventh edition of a classic primer of medical education. This book, known to all as the "Baby Robbins," has consistently matured with each past edition, including the current printing. Earlier issues of this text were written for dental students and individuals in allied health fields, but with the recent evolution of medical curricula incorporating pathophysiology and clinician teaching, recent editions have been utilized by many medical schools as a primary reference for pathology and pathophysiology courses.

The new release offers many refinements over previous issues, which include more color photographs replacing black and whites and quantitatively more images of gross and microscopic pathologic processes. Tables, used by students who succinctly organize unwieldy facts and details, may be found throughout the book, with several new tables partially substituting written text found in the previous edition. Colorful diagrams, flow charts, and schematics continue to be used as devices students can employ to understand difficult concepts not visualized in gross and microscopic images or not understood in text form.

Many relatively new concepts have been included in revised chapters, such as gastrointestinal stromal tumors and relationship with CD117. The chapter on genetics and pediatric diseases has been extended and includes new topics with more expansive coverage of laboratory diagnosis of hereditary diseases. Revisions of the chapter on diseases of the skin include a teaching component, which this reviewer recommends that the authors use in all chapters of future editions. The author of this chapter provides a section at the beginning covering basic vocabulary words with definitions needed to understand basic concepts in the chapter. Early student understanding of basic terminology provides pupils a foundation to build on.

The authors have selectively introduced the integration of disease mechanisms and laboratory diagnosis of pathologic processes. The chapter on cardiovascular disease successfully covers the laboratory diagnosis of "suspected myocardial infarction." As an attractive addition to this text, the laboratory diagnosis of diseases selectively fills a void in prior basic pathology textbooks used for medical student teaching.

In summary, this is a current, succinct, and easily comprehensible text, which covers the essence of human pathology. Medical students and course directors will certainly appreciate the new edition. The authors should be commended on the continued improvement of a respected teaching resource.

\section{Byron E. Crawford \\ Tulane University School of Medicine \\ New Orleans, Louisiana}

\section{Kumar V, Cotran RS, Robbins SL: Electronic Image Collection for Basic Pathology, 7th Edition, CD-ROM, W.B. Saunders, Philadelphia, 2003.}

Electronic Image Collection is designed as a teaching and learning resource to accompany Basic Pathology 7th Edition by Kumar, Cotran, and Robins. The authors are highly respected medical educators. This CD provides a complete set of textbook illustrations, photographs, and figure legends. The full-color images are displayed at the same size as they appear in the textbook. The files are saved at a resolution (96 pixels/ inch) suitable for on-screen viewing. They also may be pasted into presentations or other works consistent with the licensing agreement. The software requires a Web browser to open and Adobe Acrobat Reader for viewing. The program is easy to use, with three basic routes of navigation. "View Chapters" provides a table of contents based on the textbook. A thumbnail image accompanies each figure legend, and links are provided to full-size JPG and PDF files. The PDF files have more authentic color and are best for on-screen viewing and printing. The JPG files are useful for pasting into presentation programs, such as PowerPoint. "Get All Legends" enables you to save a complete set of figure legends for reference (Excel or PDF files). "Find Image" enables you to search the entire set of figure legends for topics of interest, e.g., typing in "aneurysm” pulled up six images related to this topic. Navigation arrows are conveniently located on each page, enabling you to jump quickly between chapters. The program also includes a "Lightbox" feature, which enables you to tag selected images for viewing as a group at a later time. Medical educators will find this CD a treasure trove of visual information for teaching basic and systemic pathology. Medical students will find this CD a helpful resource for class study and USMLE review. I highly recommend this beautiful, electronic image collection for all those interested in pathology education.

Bruce A. Fenderson

Jefferson Medical College

Philadelphia, Pennsylvania 